MILESTONE 25

\section{Seeing in a flash}

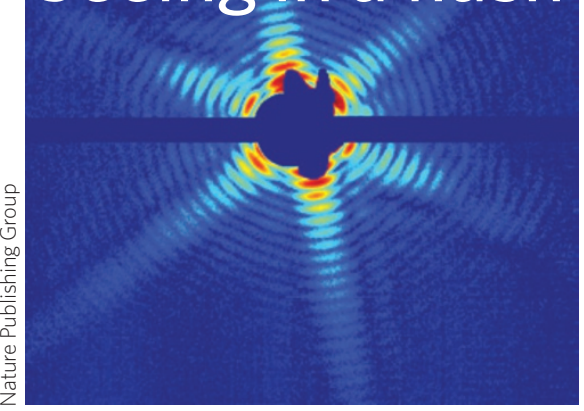

As its name suggests, crystallography generally requires crystals. Their periodic structure produces Bragg peaks in the X-ray diffraction pattern and these peaks encode and amplify the information about the underlying molecular structure. Unfortunately, many biochemical samples simply can't be crystallized sufficiently to give usable diffraction patterns. In the 1950s, drawing on earlier concepts, David Sayre noticed that a diffraction pattern contained more information than just these peaks, which could be used to reconstruct an image from only a small number of samples, provided enough signal could be captured (Milestone 7). Applying this principle to objects as small as proteins demanded a whole new approach and a whole new light source: the $\mathrm{X}$-ray free-electron laser (XFEL).

In FELs, a highly compressed electron bunch travelling through a periodic magnet array generates photons, which interact back with the electrons, causing microstructuring of the bunch. These microbunches then radiate coherently, creating $X$-ray pulses of unprecedented brilliance. Infrared FELs have been around for a few decades, but the higher energies of X-ray photons mean that XFELs are much more technically demanding. Yet their production of extremely bright and short pulses of radiation with ångström-scale wavelengths means their inherent advantages are enormous. Their major drawback is
Single-shot diffraction pattern from an individual mimivirus particle taken at the Linac Coherent Light Source in Stanford, California. Image reprinted with permission from M. M. Seibert Nature 470, 78-81 (2011).

that they are so bright that they obliterate most samples placed in their path.

In 2000, Janos Hajdu and colleagues showed how this seemingly intractable problem could be overcome. They calculated that a molecule exposed to an ultrashort $X$-ray pulse begins to explode on a timescale of around 10 femtoseconds. Light pulses shorter than this can thus pass through the molecule, capturing information about a practically unperturbed structure. And bright enough pulses will give rise to continuous diffraction patterns that are strong enough to be measured.

In principle, the proposed XFELs could provide enough photons to exploit Sayre's ideas, and using this 'diffraction before destruction' concept, they could be designed to have the right pulse duration at just the energies needed to image individual molecules. A proof-of-principle demonstration on a non-biological sample was carried out at the FLASH soft-X-ray FEL in Hamburg in 2006.

Imaging biological specimens, however, meant new sample delivery technologies, including aerosol sample injection methods developed for single particles and biomolecules, and liquid jets to produce continuous streams of nanocrystals. It also demanded new processing algorithms to piece together the diffraction patterns from all the randomly oriented molecules and produce a complete image. In 2011, these elements coalesced to give a three-dimensional image of photosystem I derived from snapshots of fully hydrated nanocrystals of the complex, and to produce projection images for the giant mimivirus particle captured in the gas phase. Other demonstrations have followed, and other XFEL programmes are underway in Europe and Japan.

In principle, many different structures could be imaged with XFELs, including cells and viruses, while their pulsed nature also offers the promise of creating movies of molecular processes. The short pulses and high energies also make them very appealing for atomic and condensed-matter physics researchers. With more of these light sources turning on across the globe, the future for XFEL science is looking very bright indeed.

Nicky Dean, Senior Editor, Nature Communications
ORIGINAL RESEARCH PAPERS Neutze, R., Wouts, R., van der Spoel, D., Weckert, E. \& Hajdu, J. Potential for biomolecular imaging with femtosecond X-ray pulses. Nature 406, 752-757 (2000) | Chapman, H. N. et al. Femtosecond diffractive imaging with a soft-X-ray free-electron laser. Nature Phys. 2, 839-843 (2006) | Chapman, H. N. et al. Femtosecond time-delay X-ray holography. Nature 448, 676-679 (2007) | Young, L. et al. Femtosecond electronic response of atoms to ultra-intense X-rays. Nature 446, 56-61 (2010) Chapman, H. N. et al. Femtosecond X-ray protein nanocrystallography. Nature 470, $73-77$ (2011) | Seibert, M. M. et al. Single mimivirus particles intercepted and imaged with an X-ray laser. Nature 470, 78-81 (2011) | Redecke, L. et al. Natively inhibited Trypanosoma brucei cathepsin B structure determined by using an X-ray laser. Science 339, 227-230 (2013) | Milathianaki, D. et al. Femtosecond visualization of lattice dynamics in shock-compressed matter. Science 342, 220-223 (2013) | Barends, T. R. M. et al. De novo protein crystal structure determination from X-ray free-electron laser data. Nature 505, 244-247 (2014) FURTHER READING McNeil, B. W. J. \& Thompson, N. R. X-ray free-electron lasers. Nature Photon. 4, 814-821 (2010) | Helliwell, J. R. How to solve protein structures with an X-ray laser. Science 339, 146-147 (2013) | Miller, R. J. D. Femtosecond crystallography with ultrabright electrons and X-rays: capturing chemistry in action. Science $\mathbf{3 4 3 , 1 1 0 8 - 1 1 1 6 ~ ( 2 0 1 4 ) ~ | ~ W a l d r o p , ~ M . ~ M . ~ X - r a y ~ s c i e n c e : ~ t h e ~ b i g ~ g u n s . ~}$ Nature 505, 604-606 (2014) 